\title{
Dopamine in plasma - a biomarker for myofascial TMD pain?
}

\author{
Andreas Dawson ${ }^{1,2,9^{*}}$, Niclas Stensson ${ }^{3}$, Bijar Ghafouri ${ }^{3,4}$, Björn Gerdle ${ }^{3}$, Thomas List ${ }^{2,5,9}$, Peter Svensson $6,7,8,9$ \\ and Malin Ernberg 8,9
}

\begin{abstract}
Background: Dopaminergic pathways could be involved in the pathophysiology of myofascial temporomandibular disorders (M-TMD). This study investigated plasma levels of dopamine and serotonin (5-HT) in patients with M-TMD and in healthy subjects.

Methods: Fifteen patients with M-TMD and 15 age- and sex-matched healthy subjects participated. The patients had received an M-TMD diagnosis according to the Research Diagnostic Criteria for TMD. Perceived mental stress, pain intensity (0-100-mm visual analogue scale), and pressure pain thresholds (PPT, kPa) over the masseter muscles were assessed; a venous blood sample was taken.

Results: Dopamine in plasma differed significantly between patients with M-TMD $(4.98 \pm 2.55 \mathrm{nM})$ and healthy controls $(2.73 \pm 1.24 \mathrm{nM} ; P<0.01)$. No significant difference in plasma 5 -HT was observed between the groups $(P=0.75)$. Patients reported significantly higher pain intensities $(P<0.001)$ and had lower PPTs $(P<0.01)$ compared with the healthy controls. Importantly, dopamine in plasma correlated significantly with present pain intensity $(r=0.53, n=14, P<0.05)$ and perceived mental stress $(r=0.34, n=28, P<0.05)$.

Conclusions: The results suggest that peripheral dopamine might be involved in modulating peripheral pain. This finding, in addition to reports in other studies, suggests that dopaminergic pathways could be implicated in the pathophysiology of M-TMD but also in other chronic pain conditions. More research is warranted to elucidate the role of peripheral dopamine in the pathophysiology of chronic pain.
\end{abstract}

Keywords: Temporomandibular joint disorders, Bruxism, Dopamine, 5-HT, Masseter muscle

\section{Background}

Dopamine and 5-hydroxytryptamine (5-HT) are neurotransmitters found in the central (CNS) and peripheral nervous systems, and in plasma. In the CNS, dopamine is involved in motor control, cognition, and the reward system [1], but possibly also in pain perception [2]. Primarily dopaminergic neurons synthesize dopamine in the CNS [1], while neuronal fibers, adrenal medulla, and neuroendocrine cells synthesize dopamine in the peripheral nervous system [1]. Goldstein and Holmes have observed that dopamine in plasma is primarily related to sympathetic activity; that is, rises in sympathetic activity

\footnotetext{
* Correspondence: andreas.dawson@regionostergotland.se

${ }^{1}$ Center for Oral Rehabilitation, Östergötland County Council, Linköping, Sweden

${ }^{2}$ Department of Orofacial Pain and Jaw Function, Faculty of Odontology, Malmö University, Malmö, Sweden

Full list of author information is available at the end of the article
}

increase release of precursors to dopamine and norepinephrine [3]. Rubi and Maechler report in their mini-review that dopamine in plasma are elevated in situations that involve psychological stress, muscle exercise, or hypovolemia [1].

Bruxism, "a repetitive jaw-muscle activity characterized by clenching or grinding of the teeth and/or by bracing or thrusting the mandible" [4], has been linked to disturbances in the central dopaminergic system [5], as has another motor function disorder, Parkinson's disease [6]. Aside from motor function-related disturbances, the most self-reported symptom in patients with Parkinson's disease is pain [7]. It has also been suggested that alterations in dopaminergic neurotransmission might be involved in burning mouth syndrome $[8,9]$, fibromyalgia $[10]$, and chronic orofacial pain $[8,9]$. The literature indicates that the dopaminergic system may be involved 
in central pain modulation, but the role of dopamine at the peripheral level in relation to pain is unknown.

Graven-Nielsen et al. found 5-HT involvement in pain transmission at the peripheral and central levels [11]. Several other studies have demonstrated that patients with chronic myalgia, including myofascial temporomandibular disorders (M-TMD) have significantly higher interstitial concentrations of 5-HT than healthy controls [12-16] and that high 5-HT levels are correlated with muscle pain and allodynia [14-17]. In addition, in patients with fibromyalgia, Ernberg et al. found that high levels of plasma 5-HT in relation to serum 5-HT may be associated with pain discomfort and high levels of anxiety [18].

Amongst others, autonomic and psychosocial factors have been suggested as risk factors for TMD $[19,20]$. Maixner et al. observed that TMD patients have a significantly altered autonomic function compared with healthy controls at rest and in stressful situations [20]. Thus, it is plausible that activity in the sympathetic nervous system of these patients is altered, and if dopamine in plasma is a potential indicator of such activity, they could be implicated in M-TMD.

The role of peripheral dopamine and 5-HT and their association with pain in patients with M-TMD is so far unknown. The aim of this study was to investigate plasma levels of dopamine and 5-HT in patients with M-TMD and healthy controls. Two hypotheses were tested: (i) patients with M-TMD have higher levels of dopamine and 5-HT in plasma than healthy subjects, and (ii) pain intensity and the pressure pain threshold correlate with plasma levels of dopamine and 5- $\mathrm{HT}$ in patients with M-TMD.

\section{Methods}

\section{Participants}

Fifteen patients with M-TMD (mean age: $31.8 \pm 13.4$ years) and an age- and gender-matched control group (mean age: $31.6 \pm 12.4$ years) participated in this study. Eleven females and four males participated in each group. Both groups were participating in other studies [12, 21, 22]. The control group was recruited from staff at Malmö University, while the patients were recruited among consecutive patients referred to the Department of Orofacial Pain and Jaw Function, Faculty of Odontology, Malmö University, Malmö, Sweden and, after a clinical examination, had been diagnosed with M-TMD [23]. One author (AD) examined the patients to confirm a diagnosis of M-TMD and the control group to confirm that the subjects were healthy.

The inclusion criteria for the M-TMD group were (i) age $>18$ years, (ii) a diagnosis of M-TMD pain [23], (iii) moderate or more severe pain in the masseter muscles upon palpation at the clinical examination and on the experimental day, and (iv) continuous or persistent pain in the jaw muscles for more than 6 months.
Inclusion criteria for the healthy controls were (i) age > 18 years, (ii) healthy, and (iii) no orofacial pain complaints.

Exclusion criteria for both groups were conditions that could influence pain sensitivity, such as systemic inflammatory connective tissue diseases (e.g., rheumatoid arthritis); whiplash-associated disorders; chronic widespread muscle pain conditions (e.g., fibromyalgia); neuropathic pain or neurological disorders (e.g., oromandibular dystonia); pain of dental origin; pregnancy or lactation; high blood pressure; and ongoing dental treatment. Another exclusion criterion was medication that could interfere with the blood analysis or pain sensitivity (e.g., anticoagulant treatment and analgesic consumption within 1 week before the experiment, for example, of paracetamol, NSAIDs, salicylate drugs, or opioids) or that could influence pain perception (e.g., anti-depressants or antiepileptic drugs). Exclusion criteria were assessed in each subject by taking a medical history.

\section{Ethics approval of the study}

This study was conducted at the Department of Orofacial Pain and Jaw Function at Malmö University. The guidelines of the Helsinki Declaration were followed, and the Regional Ethics Review Board at Lund University approved the study (2010/31). Before participation, subjects signed an informed-consent form and were informed that they could refrain from the study at any time without any consequences. After completion of participation, subjects were financially compensated.

\section{Study design}

The 1-h clinical examination, scheduled at 8 am or $1 \mathrm{pm}$ comprised an examination according to the RDC/TMD; collection of a venous blood sample for analysis of dopamine and 5-HT in plasma, and assessment of present pain intensity and the pressure pain threshold (PPT). Throughout the trial, subjects sat upright in a dental chair with head support. One operator (AD) conducted the examination.

\section{Blood sampling}

Venous blood samples were collected in 3-mL EDTA Vacutainers (Becton, Dickinson, and Company; Franklin Lake, NJ, USA). Directly after withdrawal, the samples were centrifuged $\left(2000 \mathrm{~g},+20{ }^{\circ} \mathrm{C}\right)$ for $10 \mathrm{~min}$ and the plasma was transferred into Eppendorf tubes and stored at $-70{ }^{\circ} \mathrm{C}$ until analysis.

\section{Assessment of pressure pain threshold, present pain intensity, and perceived mental stress}

An electronic algometer (Somedic Sales AB, Hörby, Sweden) was used to assess the PPT, defined as the amount of pressure $(\mathrm{kPa})$ needed to produce the 
slightest sensation of pain. A $1-\mathrm{cm}^{2}$ probe was applied to the lower attachment of the right masseter muscle with a constant pressure of $30 \mathrm{kPa} / \mathrm{s}$. The mean of three measurements made at 60-s intervals at the same area was calculated [24]. The reliability of PPT measurements on the masseter muscle was previously found to be acceptable [25].

A $100-\mathrm{mm}$ visual analogue scale (VAS) was used to assess pain intensity (anchor definitions: no pain and worst imaginable pain).

The 10-item perceived stress scale (PSS-10) was used to assess perceived mental stress during the past month in the participants. The PSS-10 measures to what degree various situations in life are perceived as stressful. Reliability and validity of the Swedish version of PSS-10 is good [26].

\section{Analysis of dopamine and 5-HT}

On the day of analysis, the plasma samples were thawed on ice. Evolute WCW solid phase extraction columns (25 mg) from Biotage (Uppsala, Sweden) were used to extract dopamine and 5-HT from the plasma. The samples were prepared using the manufacturer's recommended extraction protocol. After the analytes were eluted from the columns, the fractions were dried using a SpeedVac system and dissolved in the mobile phase used for high performance liquid chromatography with electrochemical detection (HPLC-EC). HPLC analyses were conducted as previously described [13].

\section{Statistics}

All statistical analyses were done using the Statistical Package for the Social Sciences for Windows (SPSS, v.20; IBM). Analyses were performed two-tailed at a significance level of $5 \%$. All variables are expressed as means and SDs unless otherwise stated. The ShapiroWilk's test was used to test the data for normality. Present pain intensity, PPT, perceived mental stress, and the levels of dopamine in plasma were normally distributed, while 5-HT levels in plasma were normally distributed after Ln-transformation. Parametric statistics were used in all statistical analyses excluding those of categorical variables or in analysis of M-TMD duration, since this variable was not normally distributed.

The Mann-Whitney $U$-test was used to determine significant differences between groups regarding duration of M-TMD (mos) and number of muscle and temporomandibular joints sites with pain on palpation.

Independent samples $t$-tests were used to investigate significant differences in age, pain intensity, PPT, perceived mental stress, and dopamine and 5-HT in plasma between groups. To test for significant correlations between pain intensity, PPT, perceived mental stress, and plasma dopamine, or 5-HT in each group, the
Pearson's correlation test (adjusted for multiple testing with Bonferroni correction) was used.

It has been suggested that plasma levels of dopamine and epinephrine are equivalent [1], so the sample size calculation was based on the mean $\pm \mathrm{SD}$ of the plasma level of epinephrine in healthy subjects, $0.337 \pm 0.106$ $\mathrm{nM}$ [27], using $\beta=0.80$ and $\alpha=0.05$. A power analysis found that 11 subjects in each group would suffice to detect a mean difference of 1 SD in dopamine level.

\section{Results}

Clinical characteristics of the study sample

Table 1 presents the clinical characteristics of the study sample.

\section{Dopamine and 5-HT in plasma}

Dopamine in plasma differed significantly between groups $(P<0.01)$, being higher for the patients with M-TMD than the healthy subjects. No significant group differences for plasma 5-HT were observed $(P=$ 0.75; Fig. 1).

\section{Present pain intensity, pressure pain threshold, and perceived mental stress}

Patients with M-TMD had significantly higher present pain intensity $(P<0.001$; Fig. 2$)$ and perceived mental stress $(P<0.05$; Fig. 3$)$ and significantly lower PPTs compared with healthy subjects $(P<0.01$; Fig. 2$)$.

\section{Correlations}

In the M-TMD group, present pain intensity correlated significantly with dopamine in plasma $(r=0.53, n=14$, $P<0.05)$, but not with 5-HT $(r=-0.02, n=14, P>0.05)$. A significant correlation was observed between dopamine in plasma and perceived mental stress for the group as a whole $(r=0.34, n=28, P<0.05)$. Present pain intensity correlated significantly with PPT $(r=-0.42$, $n=30, P<0.01)$ and perceived mental stress $(r=0.46$, $n=29, P<0.01) .5$-HT did not correlate significantly with present pain intensity $(r=0.005, n=27, P>0.05)$, PPT $(r=-0.011, n=27, P>0.05)$, or perceived mental stress $(r=0.104, n=27, P>0.05)$.

\section{Discussion}

The main findings of this study were that (i) dopamine in plasma was significantly higher in patients with $\mathrm{M}$ TMD than in healthy subjects; and correlated significantly with present pain intensity and perceived mental stress, (ii) present pain intensity correlated significantly with PPT and perceived mental stress, (iii) 5 -HT in plasma did not differ significantly between groups, and did not correlate significantly with present pain intensity, PPT, or perceived mental stress. 
Table 1 Clinical characteristics of the study sample

\begin{tabular}{|c|c|c|c|c|}
\hline & Patients with M-TMD & Controls & $P$ & Test \\
\hline \multicolumn{5}{|l|}{ Subjects } \\
\hline All & 15 & 15 & & \\
\hline Females & 11 & 11 & & \\
\hline Males & 4 & 4 & & \\
\hline Age (yrs) & $31.8 \pm 13.4$ & $31.6 \pm 12.4$ & 0.966 & Independent samples $t$-test \\
\hline Duration of M-TMD (months) & $58.6 \pm 60.3$ & $0.0 \pm 0.0$ & $<0.001$ & Mann-Whitney $\mathrm{U}$ test \\
\hline No. of muscle sites with pain on palpation (max. 20) & $14(8)$ & $2(2)$ & $<0.001$ & Mann-Whitney $\mathrm{U}$ test \\
\hline No. of TMJ sites with pain on palpation (max. 4) & $1(1)$ & $0(0)$ & $<0.001$ & Mann-Whitney $\mathrm{U}$ test \\
\hline Perceived mental stress (PSS-10) & $16.6 \pm 5.9$ & $12.1 \pm 3.6$ & $<0.05$ & Independent samples $t$-test \\
\hline Self-reported awake bruxism & 11 & 3 & & \\
\hline Self-reported sleep bruxism & 11 & 7 & & \\
\hline Blood sampling at 8 am & 8 & 8 & & \\
\hline Blood sampling at $1 \mathrm{pm}$ & 7 & 7 & & \\
\hline
\end{tabular}

Mean \pm SD, age (yrs), duration of M-TMD (mos), and perceived mental stress; median (interquartile range) number of muscle and temporomandibular joint (TMJ) sites with pain on palpation; frequency of self-reported awake and sleep bruxism, and blood sampling time, in 15 patients with M-TMD and 15 healthy subjects

Previous studies have suggested that dopaminergic neurotransmission is altered at a central level in patients with chronic pain conditions [8-10]. But the novel finding in this study that dopamine in plasma correlated significantly with present pain intensity in the masseter muscle suggests involvement of dopamine in pain modulation, at not only a central but a peripheral level as well. It is known that dopamine targets D1 and D2 receptors at the central level. Activation of the D1 receptor leads to formation of cAMP [28] and subsequent activation of protein kinase $\mathrm{A}$, which sensitizes tetrodotoxin (TTX)-resistant $\mathrm{Na}^{+}$channels [29], causing a pain response. On the other hand, activation of D2 receptors counteracts cAMP formation [28], possibly inhibiting sensitization of TTX-resistant $\mathrm{Na}+$ channels [29]. Such inhibition has been observed in animal studies where administration of D2 dopamine receptor antagonists in striatal neurons reduces pain $[30,31]$. On the other hand, however, it is believed that patients with chronic pain, such as burning mouth syndrome and atypical facial pain, exhibit higher densities of D2 receptors in the putamen compared with healthy subjects $[8,9]$. Thus, it seems that D2 receptors have both a painsuppressing and a pain-enhancing effect. Hypothetically, chronic activity in the dopaminergic system, possibly due to chronic pain, could cause these effects; in healthy individuals, the dopaminergic system is not chronically activated, so D2 receptors seem to be primarily associated with pain suppression.

An experimental animal study of Tambeli et al. demonstrated the existence of D1 receptors in the periphery [32]. The researchers [32] showed that dopamine injections provoked a nociceptive response, while the selective D1-receptor antagonist $\mathrm{SCH} 23390$ reversed this response. Similarly, 5-HT injections also created a nociceptive response that was reversed by the same D1receptor antagonist [32]. Thus, it seems that $5-\mathrm{HT}$ in the periphery provokes a release of dopamine, which in turn targets D1 receptors, thus causing a nociceptive response. This is particularly interesting since patients

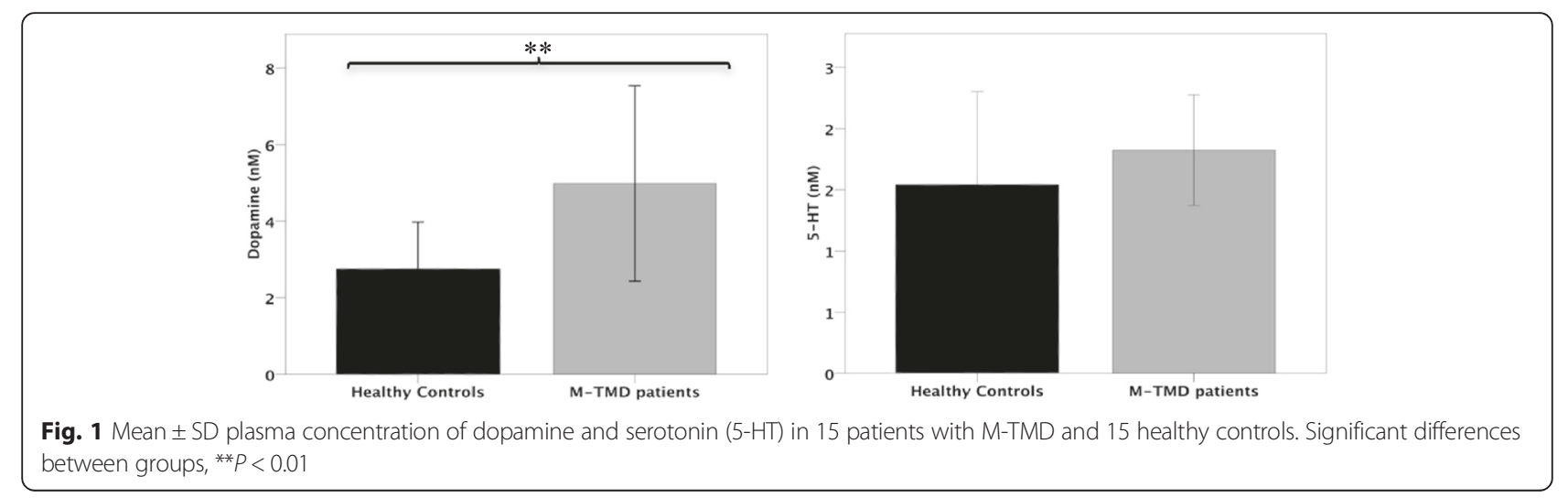



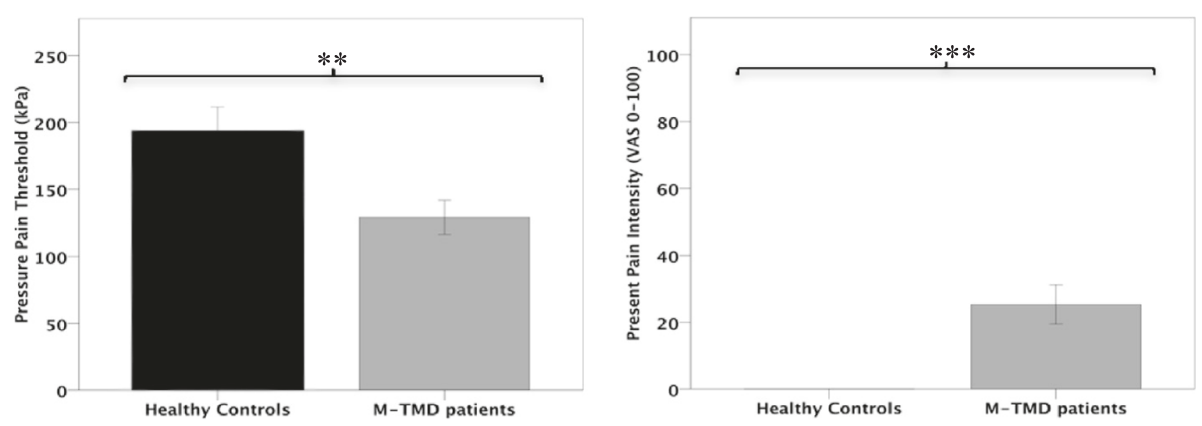

Fig. 2 Mean \pm SD pressure pain threshold ( $\mathrm{kPa}$ ), and present pain intensity (VAS 0-100) assessed in 15 patients with M-TMD and 15 healthy controls. Significant differences between groups, ${ }^{* *} P<0.01$; ${ }^{* *} P<0.001$

with M-TMD have significantly higher levels of interstitial 5-HT than healthy controls [14-16, 22]. Thus, human D1 receptors could possibly be activated by the same indirect mechanism observed by Tambeli et al. [32], contributing to the facilitation of pain. In other words, it is possible that dopamine is involved in the pathophysiology of M-TMD at a peripheral level, especially since dopamine in plasma was significantly correlated with present pain intensity.

The patients with M-TMD reported sleep bruxism and awake bruxism more often than the healthy subjects. One thought is that these bruxism habits influenced the results of the present study, since bruxism has been linked to the central dopaminergic system [5]. This is unlikely, however, since central dopamine do not pass the blood-brain barrier and is therefore differed from peripheral dopamine [1].

Circadian variation in plasma dopamine levels has been reported in humans [33], and could influence the results. In the present study, equal numbers of patients

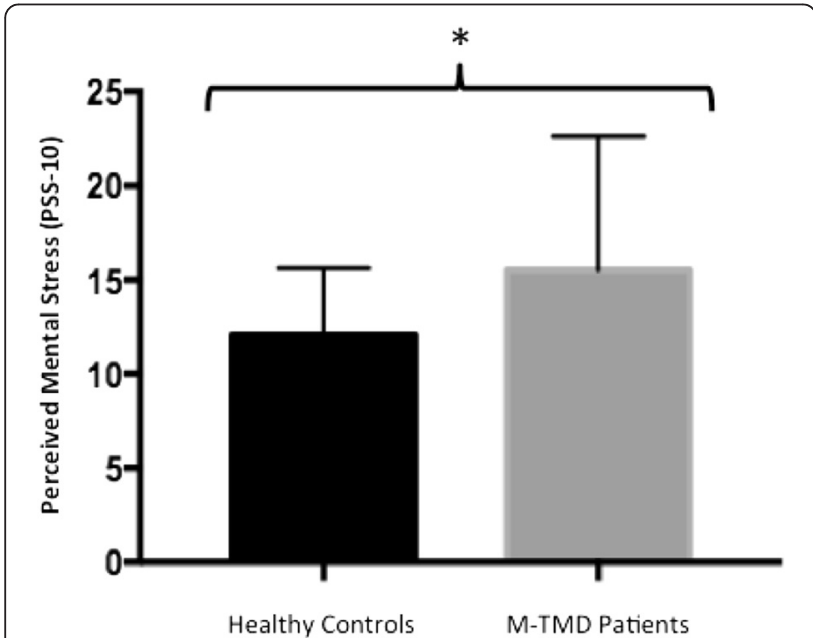

Fig. 3 Mean \pm SD perceived mental stress assessed in 15 patients with M-TMD and 15 healthy controls. Significant differences between groups, ${ }^{*} P<0.05$ with M-TMD and healthy subjects were scheduled at 8 am and $1 \mathrm{pm}$. The influence of circadian variation on plasma dopamine levels in this study should thus be minimal.

The hypothesis that patients with M-TMD would have significantly higher levels of 5-HT in plasma than healthy subjects was not confirmed. Ernberg et al. showed that a high level of 5-HT in plasma relative to in serum was significantly correlated with pain in patients with fibromyalgia [18] and that serum levels of 5-HT were significantly correlated with allodynia in patients with M-TMD [14-16]. It must be emphasized that blood plasma analyses measure the free fraction of 5-HT, while serum analyses reflect the total amount of 5-HT. Patients with M-TMD have higher levels of interstitial 5$\mathrm{HT}$ in the masseter muscles compared with healthy controls [22], thus it was hypothesized that the free fraction of 5-HT (plasma) would be significantly higher in these patients, due to an increased release from the thrombocytes, but this was not confirmed and needs to be further investigated. One explanation for these conflicting results may be that the patients with M-TMD in our study had localized pain in the masticatory muscles, while in the Ernberg et al. study [18], the patients had wide spread pain, that is, fibromyalgia.

In the present study, patients with M-TMD had significantly higher levels of perceived mental stress than healthy controls, which is in line with findings from other studies [34, 35]. We also found that perceived mental stress correlated significantly with present pain intensity and dopamine in plasma. A likely possibility is that M-TMD pain is a potential stressor. This may lead to mental stress and activation of the sympathetic nervous system (i.e., two factors that are reported to be risk factors for M-TMD pain [20,36] with subsequent peripheral release of dopamine. Measuring heart rate variability is one way to assess sympathetic activity [37], but this was not done in the present study. On the other hand, dopamine in plasma is mainly regulated by activity in the sympathetic nerves [3]. The results of the present 
study showed a significant correlation between dopamine in plasma and perceived mental stress, which supports the suggestion that peripheral dopamine is mainly regulated by sympathetic activity [3].

We observed that the patients with M-TMD had significantly lower PPTs in the masseter muscle than healthy controls, which is in line with the results of several other studies (e.g., [38, 39]. This further confirms the generally accepted knowledge that, apart from central mechanisms, peripheral mechanisms are involved in the pathophysiology of M-TMD [40]. 5-HT is involved in peripheral sensitization, and the level of interstitial 5 -HT is significantly increased in M-TMD patients compared with healthy controls [14-16, 22]. It is believed that 5-HT may provoke a peripheral dopamine release [32] that can facilitate a pain response by sensitizing tetrodotoxin (TTX)-resistant $\mathrm{Na}^{+}$channels [29]. Therefore, it is possible that peripheral dopamine contributes to peripheral sensitization, and could be an explanation for the lower PPTs observed in M-TMD patients compared with the healthy controls.

A limitation of the study is that the participants not were asked about present perceived mental stress in the experimental session. Sex hormones can alter pain levels [41], and a limitation is that female participants were not queried about menstrual cycle phase. It is, however, very likely that the females were in different phases of the menstrual cycle, which would negate any effects. A strength of this study is that the present findings are the first to highlight that peripheral dopamine could be involved in peripheral pain modulation.

\section{Conclusions}

In conclusion, the novel finding of the present study that peripheral dopamine may be involved in peripheral pain modulation means (indicates) that peripheral dopamine could be implicated in the pathophysiology of M-TMD and other chronic pain conditions. But, whether the increased level of peripheral dopamine is a consequence of pain or mental stress is not known. More research is needed to further understand the role and mechanisms of peripheral dopamine in chronic pain and mental stress.

\section{Competing interests}

The authors declare that they have no competing interests.

\section{Authors' contributions}

AD conducted the experimental sessions, performed the statistical analyses and drafted the manuscript. NS and B Ghafouri performed the chemical analyses and drafted the section about "Analysis of dopamine and 5-HT". B Gerdle, TL, PS provided with expertize and in the designing and drafting the manuscript. ME conceived the study and was involved in the statistical analyses and drafting the manuscript. All co-authors discussed the results, commented on the manuscript, and approved the final version of the manuscript.

\section{Acknowledgments}

This study was supported by grants from the Faculty of Odontology at Malmö University and the Swedish Dental Society.

\section{Author details \\ ${ }^{1}$ Center for Oral Rehabilitation, Östergötland County Council, Linköping, Sweden. ${ }^{2}$ Department of Orofacial Pain and Jaw Function, Faculty of Odontology, Malmö University, Malmö, Sweden. ${ }^{3}$ Rehabilitation Medicine, Department of Medicine and Health Sciences, Faculty of Health Sciences, Linköping University and Pain and Rehabilitation Centre, Östergötland County Council, Linköping, Sweden. ${ }^{4}$ Occupational and Environmental Medicine, Department of Clinical and Experimental Medicine, Faculty of Health Sciences, Linköping University and Centre of Occupational and Environmental Medicine, Östergötland County Council, Linköping, Sweden. ${ }^{5}$ Department of Rehabilitation Medicine, Skåne University Hospital, Lund, Sweden. ${ }^{6}$ Section of Clinical Oral Physiology, Department of Dentistry, Aarhus University, Aarhus, Denmark. ${ }^{7}$ Center for Functionally Integrative \\ Neuroscience (CFIN), MindLab, Aarhus University Hospital, Aarhus, Denmark. ${ }^{8}$ Section of Orofacial Pain and Jaw Function, Department of Dental Medicine, Karolinska Institutet, Huddinge, Sweden. ${ }^{9}$ Department of Dental Medicine, Karolinska Institutet, Huddinge, Sweden; Faculty of Odontology Malmö University, Malmö Sweden; Department of Dentistry, Aarhus University, Aarhus, Denmark.}

Received: 9 April 2016 Accepted: 27 June 2016

Published online: 07 July 2016

\section{References}

1. Rubi B, Maechler P (2010) Minireview: new roles for peripheral dopamine on metabolic control and tumor growth: let's seek the balance. Endocrinology 151(12):5570-5581. doi:10.1210/en.2010-0745

2. Leknes S, Tracey I (2008) A common neurobiology for pain and pleasure. Nat Rev Neurosci 9(4):314-320. doi:10.1038/nrn2333

3. Goldstein DS, Holmes C (2008) Neuronal source of plasma dopamine. Clin Chem 54(11):1864-1871. doi:10.1373/clinchem.2008.107193

4. Lobbezoo F, Ahlberg J, Glaros AG, et al (2012) Bruxism defined and graded: an international consensus. J Oral Rehabil. doi: 10.1111/joor.12011.

5. Lobbezoo F, Naeije M (2001) Bruxism is mainly regulated centrally, not peripherally. J Oral Rehabil 28(12):1085-1091

6. Strange PG (1993) Dopamine receptors in the basal ganglia: relevance to Parkinson's disease. Mov Disord 8(3):263-270. doi:10.1002/mds.870080303

7. Wasner G, Deuschl G (2012) Pains in Parkinson disease-many syndromes under one umbrella. Nat Rev Neurol 8(5):284-294. doi:10.1038/nrneurol. 2012.54

8. Hagelberg N, Forssell H, Aalto S, et al (2003a) Altered dopamine D2 receptor binding in atypical facial pain. Pain 106(1-2):43-48.

9. Hagelberg N, Forssell H, Rinne JO, et al (2003b) Striatal dopamine D1 and D2 receptors in burning mouth syndrome. Pain 101(1-2):149-154.

10. Wood PB, Patterson JC 2nd, Sunderland JJ et al (2007) Reduced presynaptic dopamine activity in fibromyalgia syndrome demonstrated with positron emission tomography: a pilot study. J Pain 8(1):51-58

11. Graven-Nielsen T, Arendt-Nielsen L, Mense S (2008) Fundamentals of Musculoskeletal Pain. IASP Press, Seattle

12. Dawson A, Ljunggren L, Ernberg M, et al (2014b) Effect of experimental tooth clenching on the release of beta-endorphin. J Oral Facial Pain Headache 28(2):159-164. doi:10.11607/ofph.1210.

13. Ghafouri B, Larsson BK, Sjors A et al (2010) Interstitial concentration of serotonin is increased in myalgic human trapezius muscle during rest, repetitive work and mental stress - an in vivo microdialysis study. Scand J Clin Lab Invest 70(7):478-486. doi:10.3109/00365513.2010.511257

14. Ernberg M, Lundeberg T, Kopp S (2000) Pain and allodynia/hyperalgesia induced by intramuscular injection of serotonin in patients with fibromyalgia and healthy individuals. Pain 85(1-2):31-9

15. Ernberg M, Hedenberg-Magnusson B, Alstergren $P$, et al (1999a) The level of serotonin in the superficial masseter muscle in relation to local pain and allodynia. Life Sci 65(3):313-325

16. Ernberg $M$, Hedenberg-Magnusson B, Alstergren $P$, et al (1999b) Pain, allodynia, and serum serotonin level in orofacial pain of muscular origin J Orofac Pain 13(1):56-62

17. Rosendal L, Larsson B, Kristiansen J et al (2004) Increase in muscle nociceptive substances and anaerobic metabolism in patients 
with trapezius myalgia: microdialysis in rest and during exercise. Pain 112(3):324-334. doi:10.1016/j.pain.2004.09.017

18. Ernberg M, Voog U, Alstergren P et al (2000) Plasma and serum serotonin levels and their relationship to orofacial pain and anxiety in fibromyalgia. J Orofac Pain 14(1):37-46

19. Fillingim RB, Ohrbach R, Greenspan JD et al (2011) Potential psychosocial risk factors for chronic TMD: descriptive data and empirically identified domains from the OPPERA case-control study. J Pain 12(11 Suppl):T46-T60. doi:10.1016/j.jpain.2011.08.007

20. Maixner W, Greenspan JD, Dubner R et al (2011) Potential autonomic risk factors for chronic TMD: descriptive data and empirically identified domains from the OPPERA case-control study. J Pain 12(11 Suppl):T75-T91. doi:10.1016/j.jpain.2011.09.002

21. Dawson A, Ghafouri B, Gerdle B et al (2013) Pain and intramuscular release of algesic substances in the masseter muscle after experimental tooth-clenching exercises in healthy subjects. J Orofac Pain 27(4):350-360. doi:10.11607/jop.1170

22. Dawson A, Ghafouri B, Gerdle B, et al (2014a) Effects of experimental tooth clenching on pain and intramuscular release of 5-HT and glutamate in patients with myofascial TMD. Clin J Pain 31(8):740-9

23. Dworkin SF, LeResche L (1992) Research diagnostic criteria for temporomandibular disorders: review, criteria, examinations and specifications, critique. J Craniomandib Disord 6(4):301-355

24. Svensson P, Arendt-Nielsen L, Nielsen $\mathrm{H}$ et al (1995) Effect of chronic and experimental jaw muscle pain on pain-pressure thresholds and stimulus-response curves. J Orofac Pain 9(4):347-356

25. List T, Helkimo M, Falk G (1989) Reliability and validity of a pressure threshold meter in recording tenderness in the masseter muscle and the anterior temporalis muscle. Cranio 7(3):223-229

26. Nordin M, Nordin S (2013) Psychometric evaluation and normative data of the Swedish version of the 10-item perceived stress scale. Scand J Psychol 54(6):502-507. doi:10.1111/sjop.12071

27. Goldstein DS, Feuerstein G, lzzo JL Jr et al (1981) Validity and reliability of liquid chromatography with electrochemical detection for measuring plasma levels of norepinephrine and epinephrine in man. Life Sci 28(5):467-475

28. Missale C, Nash SR, Robinson SW et al (1998) Dopamine receptors: from structure to function. Physiol Rev 78(1):189-225

29. Galbavy W, Safaie E, Rebecchi MJ et al (2013) Inhibition of tetrodotoxinresistant sodium current in dorsal root ganglia neurons mediated by D1/D5 dopamine receptors. Mol Pain 9:60-8069-9-60. doi:10.1186/1744-8069-9-60

30. Ben-Sreti MM, Gonzalez JP, Sewell RD (1983) Differential effects of SKF 38393 and LY 141865 on nociception and morphine analgesia. Life Sci 33(Suppl 1):665-668

31. Magnusson JE, Fisher K (2000) The involvement of dopamine in nociception: the role of $D(1)$ and $D(2)$ receptors in the dorsolateral striatum. Brain Res 855(2):260-266

32. Tambeli $\mathrm{CH}$, Oliveira MC, Clemente JT et al (2006) A novel mechanism involved in 5-hydroxytryptamine-induced nociception: the indirect activation of primary afferents. Neuroscience 141(3):1517-1524

33. Sowers JR, Vlachakis N (1984) Circadian variation in plasma dopamine levels in man. J Endocrinol Invest 7(4):341-345

34. Jasim H, Louca S, Christidis N et al (2014) Salivary cortisol and psychological factors in women with chronic and acute oro-facial pain. J Oral Rehabil 41(2):122-132. doi:10.1111/joor.12118

35. Lambert CA, Sanders A, Wilder RS et al (2014) Chronic HPA axis response to stress in temporomandibular disorder. J Dent Hyg 88(Suppl 1):5-12

36. Fillingim RB, Ohrbach R, Greenspan JD et al (2013) Psychological factors associated with development of TMD: the OPPERA prospective cohort study. J Pain 14(12 Suppl):T75-T90. doi:10.1016/j.jpain.2013.06.009

37. Pomeranz B, Macaulay RJ, Caudill MA et al (1985) Assessment of autonomic function in humans by heart rate spectral analysis. Am J Physiol 248(1 Pt 2): $\mathrm{H} 151-\mathrm{H} 153$

38. Farella M, Michelotti A, Steenks MH et al (2000) The diagnostic value of pressure algometry in myofascial pain of the jaw muscles. J Oral Rehabil 27(1):9-14

39. Santos Silva RS, Conti PC, Lauris JR et al (2005) Pressure pain threshold in the detection of masticatory myofascial pain: an algometer-based study. J Orofac Pain 19(4):318-324

40. Benoliel R, Svensson P, Heir GM et al (2011) Persistent orofacial muscle pain Oral Dis 17(Suppl 1):23-41. doi:10.1111/j.1601-0825.2011.01790.x

41. LeResche L, Mandl L, Sherman JJ et al (2003) Changes in temporomandibular pain and other symptoms across the menstrual cycle. Pain 106(3):253-261

\section{Submit your manuscript to a SpringerOpen ${ }^{\circ}$ journal and benefit from:}

- Convenient online submission

- Rigorous peer review

- Immediate publication on acceptance

- Open access: articles freely available online

- High visibility within the field

- Retaining the copyright to your article

Submit your next manuscript at $\gg$ springeropen.com 